\title{
Third-Order Optical Nonlinearities of Novel Phthalocyanines and Related Compounds
}

\author{
Zhongyu Li1,2, Zihui Chen², Song $\mathrm{Xu}^{1}$, Xinyu Zhou ${ }^{2}$ and Fushi Zhang ${ }^{2}$ \\ ${ }^{1}$ Changzhou University, Changzhou \\ ${ }^{2}$ Tsinghua University, Beijing
}

PR China

\section{Introduction}

The field of nonlinear optics has been developing for a few decades as a promising field with important applications in the domain of optoelectronics and photonics. Materials that exhibit nonlinear optical (NLO) behavior are useful because they allow manipulation of the fundamental properties of laser light beams, and are hence of great technological importance in areas such as photonic switching, optical computing and other optical data processing systems (Perry et al., 1994, 1996; Shirk et al., 2000). NLO activity was first found in inorganic crystals (Zyss, 1994), such as $\mathrm{LiNbO}_{3}$, but the choice of these materials is rather limited. Also, most of them have either low NLO responses or important drawbacks for processing into thin films and being incorporated into micro-optoelectronic devices. By the mid-1980s, organic materials emerged as important targets of choice for nonlinear optical applications because they exhibit large and fast nonlinearities and are, in general, easy to process and integrate into optical devices. Moreover, organic compounds offer the advantage of tailorability: a fine-tuning of the NLO properties can be achieved by rational modification of the chemical structure. Finally, they are ideal to achieve the ultimate goal of device miniaturization by going into the molecular level.

Large optical nonlinearities in organic molecules usually arise from highly delocalized $\pi$ electron systems. During the last two decades, phthalocyanines (Pcs) have been intensively investigated for their third-order NLO properties both in solutions and as thin films because of their extensively delocalized two dimensional $18 \pi$-electron system (Ho, 1987; Shirk, 1989; Kambara, 1996; Ma, 2003; Zhou, 2004; He, 2007). They also exhibit other additional advantages, namely, exceptional stability, versatility, and processability features. The architectural flexibility of phthalocyanines is well exemplified by the large number of metallic complexes described in the literature, as well as by the huge variety of substituents that can be attached to the phthalocyanine core. Furthermore, some of the four isoindole units can be formally replaced by other heterocyclic moieties, giving rise to different phthalocyanine analogues. All these chemical variations can alter the electronic structure of the macrocyclic core, and therefore, they allow the fine-tuning of the nonlinear response.

Aside from their practical interest, Pc-related molecules present very attractive features for fundamental NLO studies. Since the unsubstituted and many substituted compounds are planar (2D, two dimensional), they offer the possibility of investigating of the role of 
dimensionality on the NLO response. Moreover, by introducing peripheral substituents or by adding axial substituents, one may obtain three-dimensional (3D) structures with pyramidal shape and examine the effect of a third-dimension on the NLO response. Owing to the extended $\pi$ system, it is well-known that these Pc-related compounds exhibit a high aggregation tendency, and the aggregates usually display outstanding nonlinear optical properties. In order to improve the nonlinear optical properties of materials, the third-order optical nonlinearities of novel phthalocyanines and related compounds continue attracting attention.

This chapter arises from the need to compile the important advances obtained in the field of the NLO properties of phthalocyanines and analogues. Far from giving an exhaustive description of all the work that have been done in this area. We will focus on the third-order optical nonlinearities of some novel phthalocyanines and related compounds which were studied by our group using femtosecond degenerate four-wave mixing (DFWM) technique or picosecond Z-scan method.

\section{Theory of nonlinear optics}

Nonlinear optics is a material phenomenon in which intense light induces a nonlinear response in a medium, and in return the medium modifies the optical fields in a nonlinear way. In fact, all media are nonlinear to a certain degree of an applied optical field. The effect of a light wave on a material is usually described through the induced electrical polarization $\boldsymbol{P}$. At lower irradiation intensities, one can assume that this polarization is a linear function of the applied electric field $E$,

$$
\mathrm{P}=\chi^{(1)} \cdot \mathrm{E}
$$

where $\chi^{(1)}$ is the linear susceptibility. However, when the material is subjected to an intense applied electric field (a laser light), one must take into account the deviation of $\boldsymbol{P}$ from a linear dependence on $E$. Then the nonlinear polarization can be expressed by

$$
P=\chi^{(1)} \cdot E+\chi^{(2)} \cdot E E+\chi^{(3)} \cdot E E E+\ldots
$$

where $\chi^{(2)}$ and $\chi^{(3)}$ the quadratic (first-order) and cubic (second-order) susceptibilities, respectively. These two parameters determine the magnitude of the second- and third-order nonlinear optical responses. At the molecular level, a similar equation can be written for the microscopic polarization $\boldsymbol{P}$ induced in an atom or a molecule,

$$
P=\alpha \cdot E+\beta \cdot E E+\gamma \cdot E E E+\ldots
$$

with the coefficients $\alpha, \beta$, and $\gamma$ being the linear polarizability, the first (quadratic) hyperpolarizability, and the second (cubic) hyperpolarizability, respectively. The corresponding susceptibilities and hyperpolarizabilities are second rank $\left(\chi_{i j}{ }^{(1)}\right.$ or $\left.\alpha_{i j}\right)$, third rank $\left(\chi_{i j k}(2)\right.$ or $\left.\beta_{i j k}\right)$, and fourth rank $\left(\chi_{i j k l}(3)\right.$ or $\left.\gamma_{i j k l}\right)$ tensors, respectively. In general, geometrical symmetries may reduce the number of independent nonzero components. The odd rank tensor $\chi^{(2)}$ is zero in centrosymmetric media whereas the even rank tensor $\chi^{(3)}$ does not have any symmetry restrictions and can take place in any materials. $\chi^{(\mathrm{n})}$ is frequency dependent and, as a result, resonant and non-resonant parameters differ significantly depending upon the measurement frequencies. The macroscopic third-order 
susceptibility $\chi^{(3)}$ is directly related to the microscopic averaged hyperpolarizability $\gamma\left(-\omega_{4} ; \omega_{1}, \omega_{2}, \omega_{3}\right)$ through local field correction factors $f\left(\omega_{i}\right)$ at the frequencies of the applied electric fields through the relation,

$$
\chi^{(3)}\left(-\omega_{4} ; \omega_{1}, \omega_{2}, \omega_{3}\right)=N f\left(\omega_{4}\right) f\left(\omega_{1}\right) f\left(\omega_{2}\right) f\left(\omega_{3}\right) \gamma\left(-\omega_{4} ; \omega_{1}, \omega_{2}, \omega_{3}\right)
$$

where $\omega_{4}$ is the output frequency, $\omega_{1}, \omega_{2}$ and $\omega_{3}$ are the input ones, relating with each other by the expression $\omega_{4}=\left|\omega_{1} \pm \omega_{2} \pm \omega_{3}\right| ; N$ is the number of molecules per volume unit which can be obtained from the product of mass density $D$ and Avogadro's constant $A$, divided by the molar mass $M$, i.e., $N=A \times D / M ; f\left(\omega_{i}\right)$ is the local field factor at irradiation frequency $\omega_{i}$. The local field factor at optical frequencies for a pure liquid can be estimated by the expression derived by Lorentz

$$
\left.f\left(\omega_{i}\right)=\left[n\left(\omega_{i}\right)^{2}+2\right)\right] / 3
$$

where $n\left(\omega_{i}\right)$ is the linear refractive index for a liquid at frequency $\omega_{i}$. The local field is the actual electric field acting on the microscopic species in the material. It should be emphasized that Lorentz's local field factor is an approximation since it considers the species to occupy a spherical cavity in the material and the local environment of the species is treated as a continuum. Also note that the indices of refraction used in the local field approximations for optical frequencies are generally assumed to be independent of the applied electric field.

\section{Measurement of third-order NLO properties}

The initial stages in the development of novel third-order NLO materials are the synthesis of molecules with large $\gamma$ value and their incorporation into materials with substantial $\chi^{(3)}$ coefficient. Although many additional factors must be considered for practical device applications (e.g., thermal and photochemical stability, processability, etc.) the NLO properties of molecules and molecular materials can be adequately described here by reference to values of $\gamma$ and $\chi^{(3)}$. Each of two parameters can be measured by using various experimental techniques. In most such measurements, the effects of resonance must always be considered; $\gamma$ and $\chi^{(3)}$ are all wavelength dependent, being strongly resonance enhanced whenever the fundamental or harmonic frequencies are close to an electronic excitation. Although large NLO responses are required, any significant absorption of light is obviously highly undesirable for most application of NLO materials. This aspect has led to the use of expression "transparency/efficiency trade-off" when discussing the merit of such materials.

For studies of cubic NLO properties, values of $\gamma$ and $\chi^{(3)}$ are usually obtained from thirdharmonic generation (THG) (Hermann et al., 1973) or degenerate four-wave mixing (DFWM) (Linder et al., 1982). The electric-field induced second-harmonic generation (EFISHG) technique may also be used to determine $\gamma$. THG studies simply involve measurement of the light produced at the third harmonic (TH), but are often complicated by the fact that many NLO materials absorb strongly in the TH region, even when using a $1907 \mathrm{~nm}$ fundamental which gives a TH at $636 \mathrm{~nm}$. THG occurs almost instantaneously through purely electronic interactions that do not depend on the population of the excited state. The disadvantage of the THG technique is that dynamic nonlinearities are 
not probed and no information on the time response of the optical nonlinearity can be given.

DFWM is a convenient method for measuring both electronic and dynamic nonlinearities. It involves three laser beams of the same frequency interacting in a materials to produce a forth degenerate beam, the intensity of which allows determination of $\chi^{(3)}$. DFWM may hence circumvent the effects of resonance, but has the additional aspect that vibrational and orientational mechanisms can contribute substantially to the observed optical nonlinearity. In contrast, only the electronic hyperpolarizability is fast enough to be probed by THG measurements. Hence, THG-derived $\gamma$ values are generally considerably smaller than those determined via DFWM, and are more useful for deriving structure activity correlations (SACs) for the purely electronic part of $\gamma$, i.e., the only part of interest for potential practical applications. Polarization and time-dependent DFWM measurements can be used to distinguish the electronic (parametric) part of $\gamma$ from other (nonparametric) components, but several experimental subtleties must be considered if this is to be achieved reliably. It is important to remember that $\chi^{(3)}$ values which are most often determined for solution will always be concentration dependent.

Another experimental method that has become popular for characterization of cubic NLO materials is the relatively simple, but highly sensitive Z-scan technique which measures the phase change induced in a single laser beam on propagation through a material (SheikBahae et al., 1989, 1990). The sample is moved along the propagation path (z) of a focused Gaussian beam whilst its transmittance is measured through a finite aperture in the far filed, and the sign and magnitude of the nonlinear refractive index $n_{2}$ are deduced from the resulting transmittance curve (Z-scan). If the value of $n_{2}$ is positive, then the material has a tendency to shrink a laser pulse and is termed "self-focusing" (SF), whilst a negative $n_{2}$ characterized "self-defocusing" (SDF) behaviour. Although the ultrafast, electronic $n_{2}$ arises from the real part of $\chi^{(3)}$, published $n_{2}$ values often include, and indeed may be dominated by, nonparametric (thermal) contributions. In cases where nonlinear refraction is accompanied by nonlinear absorption, the nonlinear absorption coefficient $\alpha_{2}$ can be determined by performing a second Z-scan with the aperture removed. Effective $\chi^{(3)}$ and $\gamma$ values can also be determined via this method, but must be interpreted with great care in term of their actual origins which may be largely thermal effects.

Ultrafast, time-resolved optical Kerr effect (OKE) measurements (which involve another type of four-wave mixing process) may also be occasionally be used to determine $\chi^{(3)}$ and $\gamma$ values. The OKE is the optically-induced birefringence caused by a nonlinear phase shift. There are various mechanisms (electronic deformation, molecular reorientation, molecular libration, molecular redistribution, electrostriction, thermal change) that are responsible for the change of nonlinear refractive index in the OKE and each mechanism has different strengths and response times. In OKE, a strong beam is used as a pump beam and a weak beam is used as a probe beam. The intensity of the probe beam transmitted through a Kerr cell is measured as a function of the delay time between pump and probe.

In each of the THG, DFWM, Z-scan and OKE techniques, $\gamma$ is derived from the measured $\chi^{(3)}$ values by using the solute number density of the solution. In a few cases, $\chi^{(3)}$ values have also been determined by using Stark spectroscopy. It should be remembered that comparisons of $\chi^{(3)}$ or $\gamma$ values obtained using different techniques with different experimental conditions are generally of little utility. 


\section{Novel phthalocyanines and analogues with third-order NLO properties}

The third-order NLO properties of phthalocyanines and its analogues have been extensively investigated since 1990, and this area has been reviewed (Torre et al., 2004). The discussion here will be limited to selected highlights of our studies and more recent developments.

\subsection{Novel phthalocyanines}

The electronic structure of phthalocyanine molecules can be tailored by either metal substitution at the central binding site or by altering the peripheral and axial functionalities, thus affording great versatility in controlling their electro-physical properties. Incorporation of a metal atom in the center of the macrocycle results in two types of charge-transfer transitions: metal-to-ligand and ligand-to-metal. The molecular hyperpolarizability could be enhanced by the metal-ligand bonding through the transfer of the electron density between the metal atom and the conjugated ligand systems. Furthermore, peripheral substituents could also influence the nonlinearity through intermolecular interactions. Therefore, the third-order NLO susceptibility $\chi^{(3)}$ could vary by several orders of magnitude through chemical modification of the macrocycle structure (Nalwa et al., 1993, 1999; He et al., 2007).

\subsubsection{Diarylethene-phthalocyanine dyads}

In general, the absorption spectra of monomeric phthalocyanines are dominated by two intense bands, a Soret band in the near ultraviolet region (at around $350 \mathrm{~nm}$ ) and a $\mathrm{Q}$ band in the visible region (at around $670 \mathrm{~nm}$ ), with a molar extinction coefficient in the range of $10^{5} \mathrm{M}^{-1} \mathrm{~cm}^{-1}$. When electron-donating alkoxy/aryloxy groups are introduced at the periphery closest to the phthalocyanine ring, a large bathochromic shift occurs in the $Q$ band. These large red-shifted phthalocyanines are very soluble in common organic solvents and can be easily be prepared thickness-controlled thin films for practical applications to photonic devices. However, little attention has been paid to the third-order nonlinear properties of these molecules. Herein, we report the third-order nonlinear optical properties of aryloxy substituted novel diarylethene-phthalocyanine dyads 1-4 (the structures are shown in Figure 1) measured by femtosecond DFWM technique at $800 \mathrm{~nm}$ under offresonant condition ( $\mathrm{Li}$ et al., 2007).

Figure 2 shows the temporal response of the DFWM signal as a function of the probe delay in the diarylethene-phthalocyanine dyads 1-4. The DFWM signal of each sample is wellfitted by a Gaussian function (solid curve), and the half-width of fitting curve is similar to the autocorrelated pulse duration. All the signal profiles almost exhibit symmetry about the maximum signal (the zero time delay), which indicate that the responses time of the thirdorder optical nonlinearities are shorter than the experimental time resolution (50 fs). Such instantaneous response means that the Kerr effect (electronic component) from the distortion of the large $\pi$-conjugated electron charge distribution of diarylethenephthalocyanine dyad molecules is main reason for generating the DFWM signal.

The evaluated values of $\chi^{(3)}, \gamma$ and nonlinear refractive index for the diarylethenephthalocyanine dyads 1-4 in DMF solution are summarized in Table 1. Among the samples, diarylethene-phthalocyanine dyad 4 possesses the highest $\gamma_{\mathrm{e}}$ value of $1.12 \times 10^{-30} \mathrm{esu}$. It was found that the second-order hyperpolarizability values of the diarylethene-phthalocyanine 


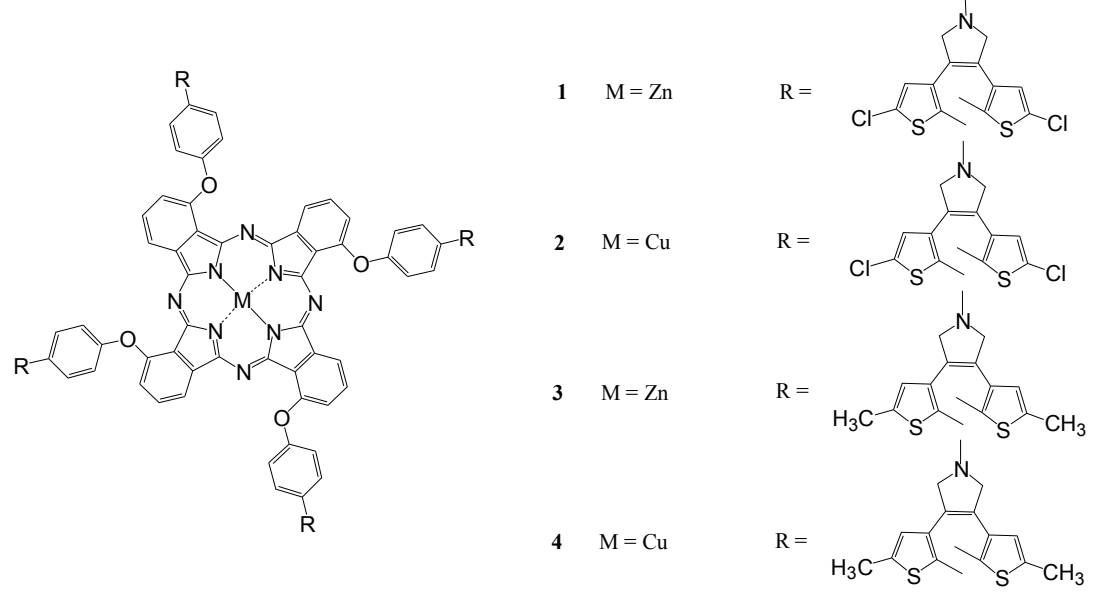

Fig. 1. Structures of diarylethene-phthalocyanine dyads 1-4
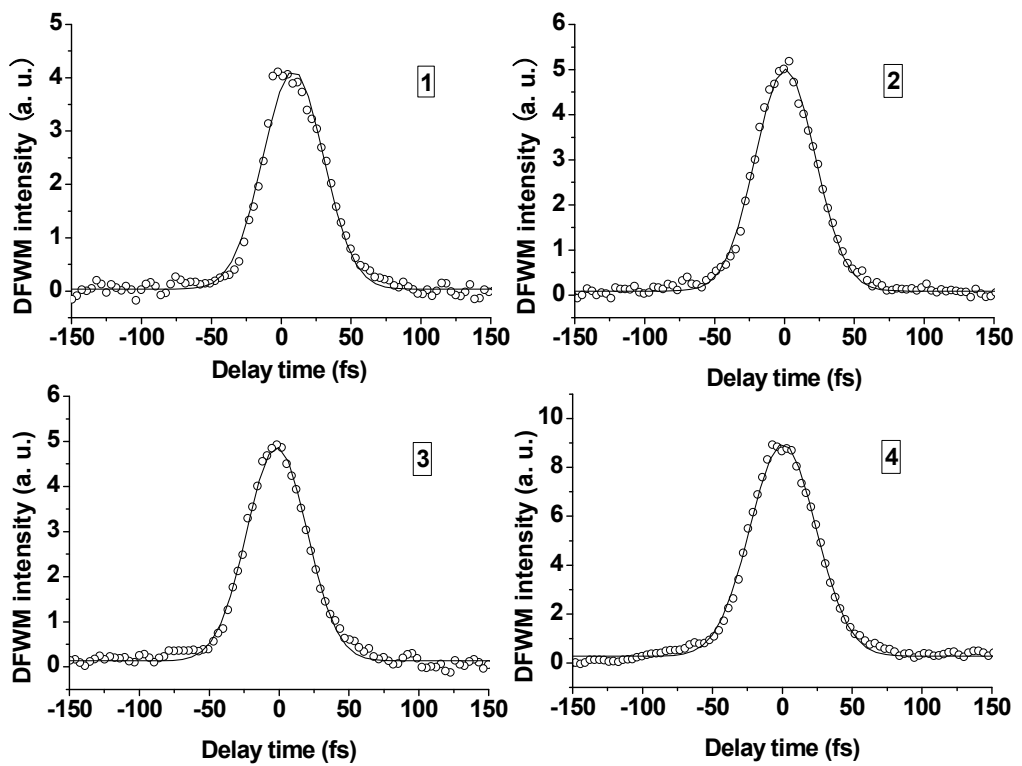

Fig. 2. Temporal profiles of DFWM signal of the diarylethene-phthalocyanine dyads 1-4

dyads 1-4 are 2-3 times larger than that of tetra-aryloxy substituted metal-free phthalocyanine. This enhancement of $\gamma$ mainly comes from the $\mathrm{d}$ valence orbital contribution of central metal atoms $(\mathrm{Zn}$ and $\mathrm{Cu}$ ) of the diarylethene-phthalocyanine dyads. Furthermore, it is observed that the $\gamma$ values of the copper substituted 
diarylethene-phthalocyanine dyads (Cu-DE-Pcs) are larger than those of the zinc substituted diarylethene-phthalocyanine dyads (Zn-DE-Pcs). This behavior can explain from the electronic structures of the upper occupied and lower vacant molecular orbitals for the ground states of metal substituted phthalocyanines (MPcs). In the case of Zn-DEPcs with completely filled d-shell, we can suppose that the probability for the charge transfer mechanism is very less. The $3 \mathrm{~d}$ subshell of Zn-DE-Pcs is filled and deep enough to form rather pure molecular orbitals. The Zn-DE-Pcs exhibit a large gap between the $\mathrm{HOMO}$ and LUMO. In the case of Cu-DE-Pcs, the unfilled d valence orbital can be split into serials level due to the interaction between the $\mathrm{d}$ electrons and $\pi$-conjugation electrons of Pc ring, this result will lower the transition energy in low-lying d orbitalligand or d-d transition. The existence of excited state with low transition energy will enhance the nonlinear optical susceptibilities of the material. The unfilled $\mathrm{d}$ orbit of $\mathrm{Cu}$ atoms will couple with the conjugated electrons of Pc ring leading to the extension of conjugated systems. As a result, the Cu-DE-Pcs with larger conjugated systems will show larger optical nonlinearities than the Zn-DE-Pcs. Moreover, the $\gamma$ value of the methyl substituted diarylethene-phthalocyanine dyad $\mathbf{3 / 4}$ is larger than that of the relative chlorine substituted diarylethene-phthalocyanine dyad $\mathbf{1 / 2}$. This is probably attributed to electron-pushing effect of the methyl group of the diarylethene-phthalocyanine dyad 3 and 4 , which leads to large polarization of molecules.

\begin{tabular}{cccc}
\hline Sample & $\chi^{(3)}\left(\times 10^{-14} \mathrm{esu}\right)$ & $\gamma\left(\times 10^{-31} \mathrm{esu}\right)$ & $n_{2}\left(\times 10^{-13} \mathrm{esu}\right)$ \\
\hline $\mathbf{1}$ & 4.50 & 7.60 & 8.33 \\
$\mathbf{3}$ & 5.05 & 8.53 & 9.35 \\
$\mathbf{4}$ & 4.92 & 8.32 & 9.12 \\
& 6.62 & 11.2 & 12.3 \\
\hline
\end{tabular}

Table 1. Evaluated values of $\chi^{(3)}, \gamma$ and nonlinear refractive index for the diarylethenephthalocyanine dyads 1-4 in DMF solution

\subsubsection{Azobenzene-phthalocyanine dyads}

In 2008, we reported the photo-responsive J-aggregation behavior of a novel $\alpha$-aryloxysubstituted zinc phthalocyanine (azobenzene substituted zinc phthalocyanine, hereafter, abbreviated as azo-ZnPc dyad) and its third-order optical nonlinearity (Chen et al., 2008). The azo-ZnPc dyad was synthesized through a rather facile route as shown in Scheme 1 . The third-order optical nonlinearities of the photo responsive J-aggregates of the azo-ZnPc dyad (before and after irradiation conditions) were measured using a Z-scan technique at $532 \mathrm{~nm}$ with pulse duration of 25 ps.

In our previous studies, it was found that $\alpha$-aryloxy-substituted Zinc phthalocyanine could form J-type self-aggregate in noncoordinating solvents through the complementary coordination of the peripheral oxygen atom of one phthalocyanine to the central $\mathrm{Zn}^{2+}$ in another phthalocyanine (Zhang et al., 2007a, 2007b). Moreover, the $\alpha$-aryloxy-substituted Zinc phthalocyanine formed J-aggregate showed another characteristic, that is the addition of methanol could break the J-aggregate, as a consequence, typical Q-band restored. 
<smiles>N#Cc1ccc(N=Nc2ccccc2)cc1Oc1ccc(N=Nc2ccccc2)c(N)c1</smiles>

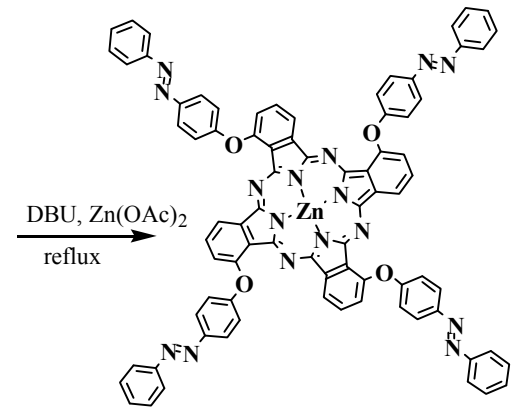

Scheme 1. Synthesis route of a novel $\alpha$-aryloxy-substituted zinc phthalocyanine

The studied azo-ZnPc dyad shows same absorption behaviors as that of the reported $\alpha$ aryloxy-substituted Zinc phthalocyanine. Figure 4 shows the spectra change of initial and UV-illuminated solutions of azo-ZnPc in chloroform with the addition of methanol. As methanol was titrated, the absorption at $740 \mathrm{~nm}$ of both of the solutions decreased gradually and finally disappeared completely with the increase in the absorption at 698 $\mathrm{nm}$. The unusual red-shifted peak at $740 \mathrm{~nm}$ can be attributed to the formation of Jaggregates. For azo-ZnPc, there is a stronger tendency to form J-aggregate when the azobenzene units is in the cis-conformation, and the irradiation of UV light will cause enhancement of J-aggregation.
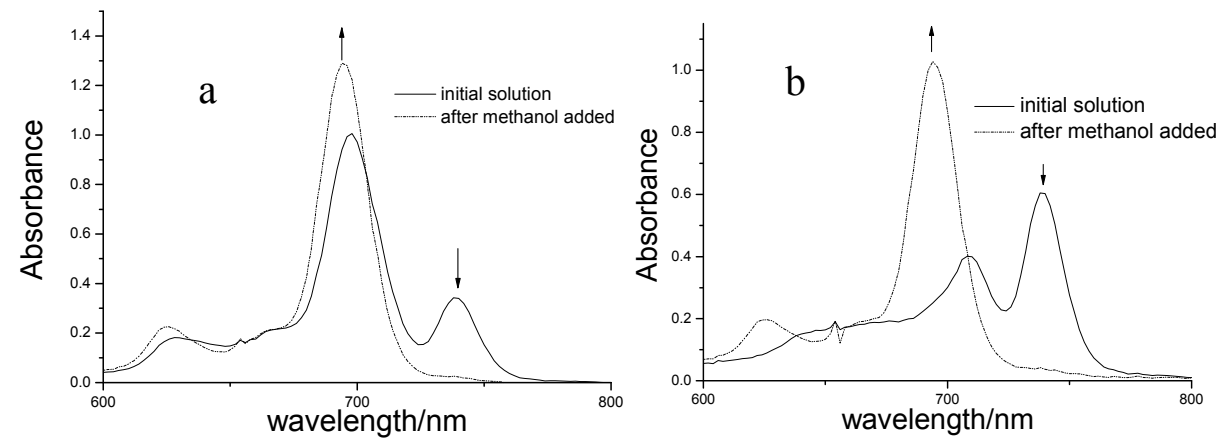

Fig. 3. Absorption spectral changes of azo-ZnPc (a: before UV light irradiation; b: after UV light irradiation for $3 \mathrm{~min}, \mathrm{c}=1.19 \times 10^{-5} \mathrm{M}$ ) in chloroform .

The open aperture curves (Figure 4) of Z-scan measurement exhibit the normalized valleys, indicating the presence of reverse saturable absorption with a positive coefficient $\beta$. And the normalized transmission for the closed aperture of Z-scan measurement is shown in Figure 5. The large valley-to-peak configurations of closed aperture curves suggest that the refractive index changes are negative, exhibiting a strong defocusing effect. 


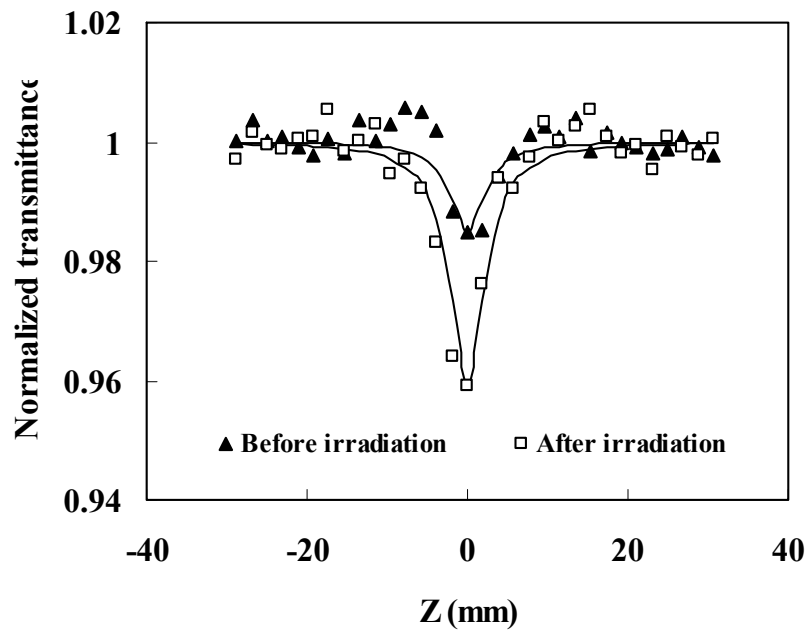

Fig. 4. Normalized transmission without aperture at $532 \mathrm{~nm}$ (open aperture) as a function of distance along the lens axis. The filled triangles and open squares are measured data for before irradiation and after irradiation of azo-phthalocyanine, respectively. Each point corresponds to the average of 5 pulses. The solid line is the theoretical fit.

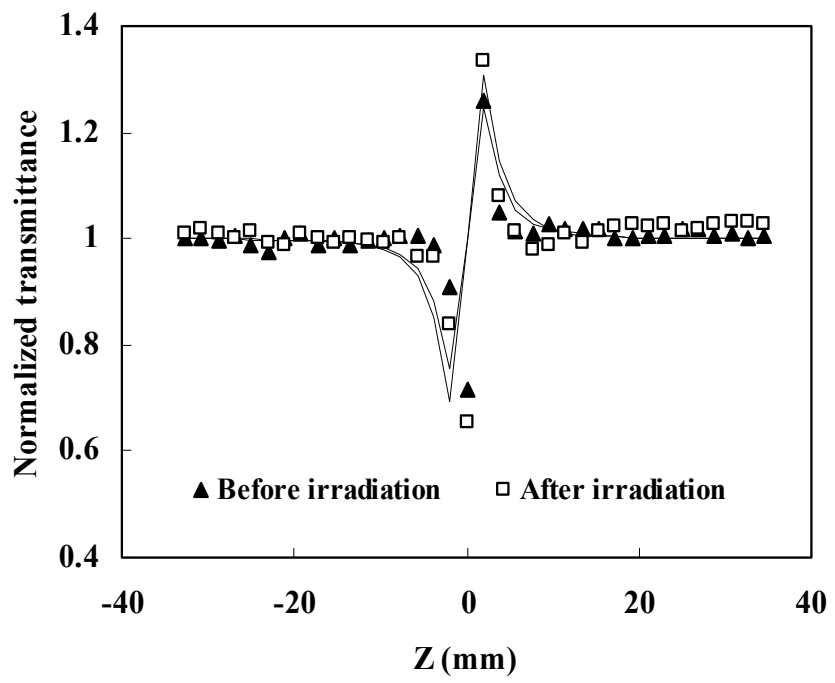

Fig. 5. Normalized transmission for the closed aperture of Z-scan measurement

The nonlinear absorption coefficient $(\beta, \mathrm{m} / \mathrm{W})$, the nonlinear refraction coefficient $\left(n_{2}\right.$, $\left.\mathrm{m}^{2} / \mathrm{W}\right)$, the third-order nonlinear susceptibility $\left(\chi^{(3)}\right.$, esu) and the molecular second hyperpolarizability $(\gamma, \mathrm{esu})$ are calculated and listed in Table 2 . It is found that the studied azo-phthalocyanine dyad shows large second-order molecular hyperpolarizabilities which are of the order of $10^{-30}$ esu both before and after irradiation conditions. The value of $\gamma$ of 
before irradiation is 1.25 times larger than that of after irradiation condition. This enhancement may be attributed to the increase of J-aggregation degree of azo-ZnPc dyad after UV light irradiation.

\begin{tabular}{ccc}
\hline Sample & Before irradiation & After irradiation \\
\hline$n_{2} / \times 10^{-19} \mathrm{~m}^{2} \mathrm{~W}^{-1}$ & 4.26 & 5.30 \\
$\beta / \times 10^{-9} \mathrm{~m} \mathrm{~W}^{-1}$ & 1.48 & 4.03 \\
$\chi^{(3)} / \times 10^{-13} \mathrm{esu}$ & 2.26 & 2.82 \\
$\gamma \gamma / \times 10^{-30} \mathrm{esu}$ & 3.87 & 4.82 \\
\hline
\end{tabular}

Table 2. Values of The nonlinear absorption coefficient, the nonlinear refraction coefficient, the third-order nonlinear susceptibility and the molecular second hyperpolarizability of Azo-ZnPc dyad.

\subsubsection{Azobenzene-containing water soluble unsymmetrical phthalocyanines}

In 2009, we reported the photoswitching of the third-order nonlinear optical properties of unsymmetrical azobenzene-containing metal phthalocyanines (structures are shown in Figure 6) based on reversible host-guest interacions (Chen, 2009).

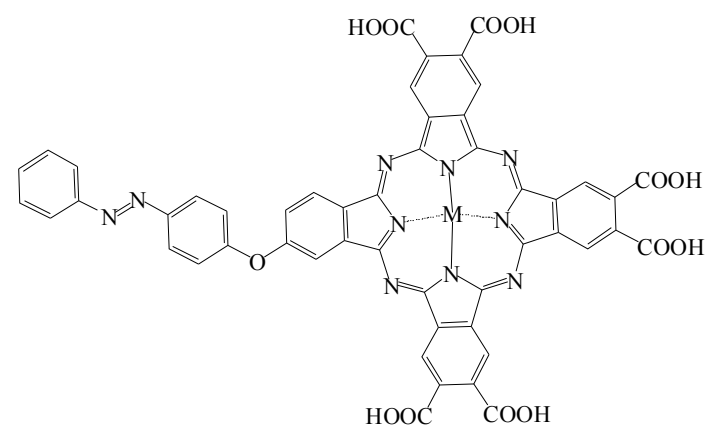

\section{MPc7}

Fig. 6. Structures of unsymmetrical azobenzene-containing metal phthalocyanines

It has been well established that azobenzene could reversibly assemble with $\alpha$-cyclodextrin through host-guest interaction under suitable external photo-stimuli, and this phenomenon has been exploited as the basis of some molecular shuttles and motors (Breslow \& Dong, 1998; Dugave \& Demange, 2003). However, their applications in phthalocyanine chemistry have rarely been studied yet. We believe that this reversible host-guest interaction can be used to modulate the NLO properties of phthalocyanines if the phthalocyanines were judicious designed. Therefore, we prepared for the first time two azobenzene containing water soluble unsymmetrical metal phthalocyanines. Their reversible host-guest interaction with $\alpha$-cyclodextrin in aqueous media and the resulting effects on the NLO properties of such molecules were also investigated. Scheme 2 shows the structures and the synthesis of target azobenzene containing water soluble unsymmetrical zinc (II) and copper (II) phthalocyanines (abbreviated as Zn-Pc7 and Cu-Pc7, respectively) and their inclusion complexes with $\alpha$-cyclodextrin (Zn-Pc8, Cu-Pc8) 

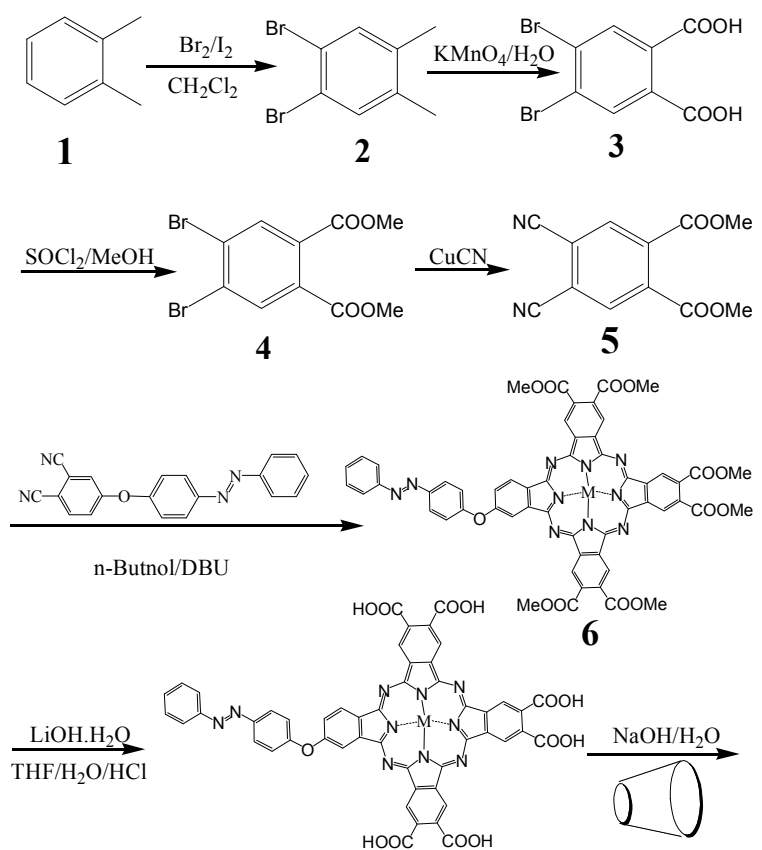

Pc7

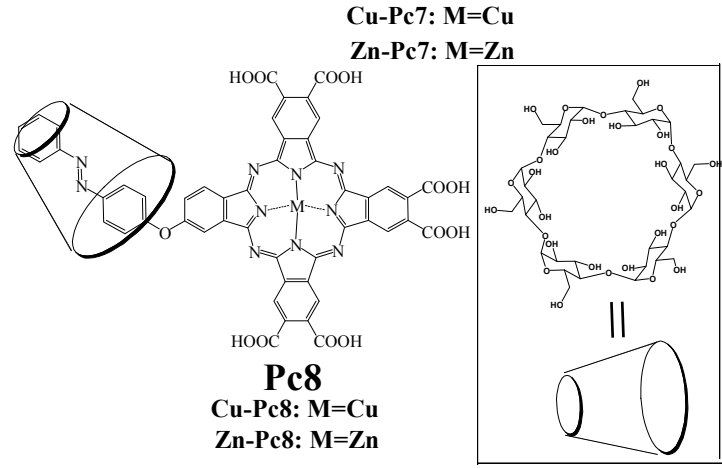

Scheme 2. Synthesis of target azobenzene containing water soluble unsymmetrical zinc (II) and copper (II) phthalocyanines and their inclusion complexes with $\alpha$-cyclodextrin (Zn-Pc8, $\mathrm{Cu}-\mathrm{Pc} 8)$

Z-scan studies show that each azobenzene containing water soluble unsymmetrical phthalocyanine consists of an electro donating phenylazophenoxy group (D) and six electron withdrawing carboxyl (A) forming a D- $\pi$-A alignment along the $\mathrm{x}$ axis. As a result of such unique chemical structure, all the samples showed very large molecular cubic hyperpolarizabilities which are of the order 10-30 esu. The Azobenzene moieties of these compounds could reversibly associate with $\alpha-C D$ to form inclusion complexes through hostguest interaction in aqueous media upon alternating illumination of $\mathrm{UV}$ and visible light, resulting apparent influences to the third-order NLO properties of these phthalocyanines. 
This influence is especially striking for the phthalocyanine whose central metal atom is $\mathrm{Cu}^{2+}$. The molecular cubic hyperpolarizability $\gamma$ of its inclusion complex with $\alpha-\mathrm{CD}$ is 2.10 $\times 10^{-30} \mathrm{esu}$. When the inclusion complex disassociated under the illumination of $365 \mathrm{~nm}$ light, the $\gamma$ value was $4.2 \times 10^{-30} \mathrm{esu}$, which is an $100 \%$ increase. Taking account of the large molecular cubic hyperpolarizabilities of these compounds, our endeavors toward ideal third-order NLO photoswitching systems is very promising, with sufficient room for improvement. This work suggested that reversibly control either the chemical structure or the molecular packing arrangement of excellent third-order NLO materials is an attractive strategy for constructing ideal third-order NLO photoswitching systems. Moreover, the present study emphasized the reversible host-guest interaction between azobenzene and $\alpha-C D$ on the packing style of phthalocyanines, which may provide new insights to the host-guest chemistry.

\subsubsection{Novel copper phthalocyanine-ferrocene dyad}

González-Cabello (González-Cabello et al., 2003) reported that the interplanar distance between the two ciclopentadiynyl rings combined with the rigid, stereochemically well defined, $\pi$-conjugated linkers between the ferrocene and the Pc-subunits provide an excellent situation for the cofacial stacking of the Pc macrocycles, thus allowing potential NLOfavorable through space interactions between the individual Pc subunits. Moreover, the combination of an electron acceptor moiety such as phthalocyanine and electron donor unit such as ferrocene may give rise to intramolecular charge transfer that may enhance the nonlinear optical response. Herein, third-order nonlinear optical property of a novel copper phthalocyanine-ferrocene dyad (the structure is shown in Figure 7) measured by femtosecond degenerate four-wave mixing technique under off-resonant condition (Bin et al., 2008).

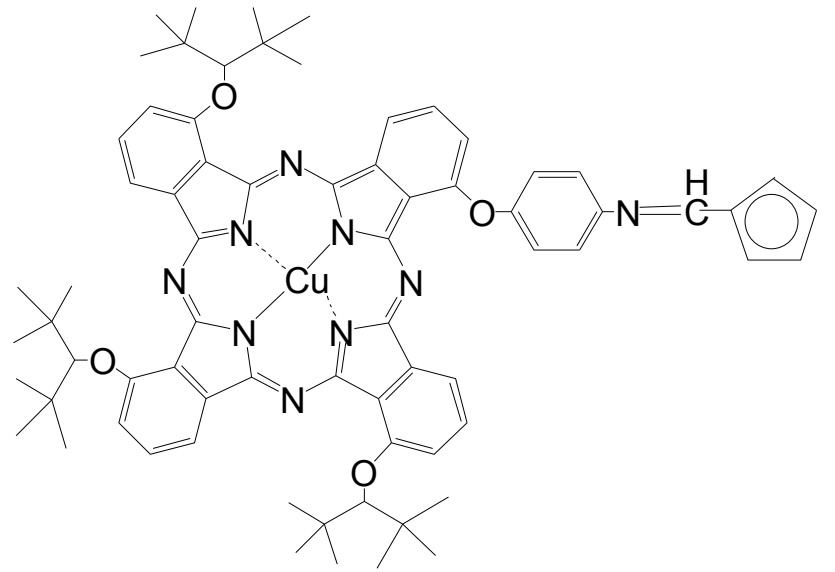

Fig. 7. Structure of novel copper phthalocyanine-ferrocene dyad

The DFWM measurement shows that the second-order molecular hyperpolarizability of this compound was measured to be $1.74 \times 10^{-30} \mathrm{esu}$, and its response time was also obtained and no more than $50 \mathrm{fs}$. This large and ultrafast third-order optical nonlinear response is mainly enhanced by the formation of intramolecular charge-transfer which can enhance the delocalized movements of the large $\pi$-electrons in the molecules. 


\subsubsection{Novel thiophene-bearing phthalocyanines}

Recently, we have measured the third-order nonlinear optical properties of two thiophenebearing phthalocyanines (Ni-TPc and $\mathrm{Cu}-\mathrm{TPc}$, their structures are shown in Figure 8) using Z-scan technique at $532 \mathrm{~nm}$ (Chen et al., 2011). Both Ni-TPc and Cu-TPc were found to show large molecular cubic hyperpolarizabilities whose values are of the order of $10^{-30} \mathrm{esu}$. The $\gamma$ value of $\mathrm{Cu}$-TPc is 1.5 times larger than that of Ni-TPc, mainly as a result of their notably different nonlinear absorptions. Most notably, the nonlinear absorption and the nonlinear refraction contribute almost equally to their molecular cubic hyperpolarizabilities, while for our previous studied Pcs, the nonlinear refraction always plays an absolutely predominant role. It is assumed that the incorporation of thiophene rings into phthalocyanines could notably increase the multi-photo absorption cross-section of Pc.

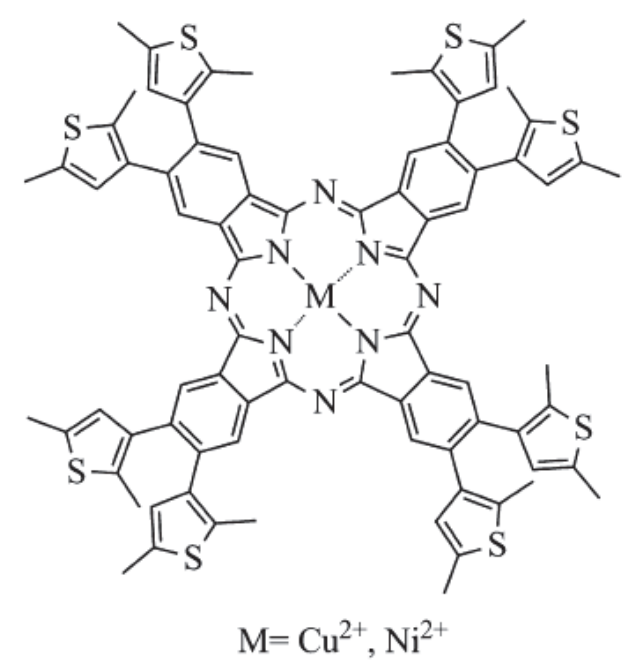

Fig. 8. Chemical structures of two thiophene-bearing phthalocyanines, Ni-TPc and Cu-TPc

\subsection{Novel phthalocyanine related compounds}

Phthalocyanines are special organic systems in a way that they offer tremendous opportunities in tailoring their photophysical and optical properties over a wide range either by substituting different metal atoms into the central binding site or by altering the peripheral and axial functionalities. It is possible to incorporate a variety of peripheral substituents around the phthalocyanines core as well as replace some of the isoindole units by other heterocyclic moieties, giving rise to different phthalocyanine analogues. For example, in the previous studie (Liu et al., 1999), it was shown that the phthalocyanine may lose a bridging nitrogen atom when complexed with phosphorus which was proved to be a phthalocyanine analogue-dihydroxy phosphorus (V) tetrabenzotriazacorrole (TBC).

\subsubsection{Dihydroxy phosphorus (V) tetrabenzotriazacorroles}

Due to its special three-dimensional $\pi$-electron structure and features which are different from phthalocyanines, the TBC macrocycle should have a potential application in photonic devices. However, to our knowledge, there is no report on third-order optical 
nonlinearity of dihydroxy phosphorus (V) tetrabenzotriazacorrole. Herein, we report the third-order nonlinear optical properties of a series phthalocyanine analogues, nonsulfonated $\left\{\mathrm{P}(\mathrm{OH})_{2} \mathrm{TBC}\right\}$, sulfonated $\left\{\mathrm{P}(\mathrm{OH})_{2} \mathrm{TBCSn}\right\}$ and isopropoxyl substituted $\left\{\mathrm{P}(\mathrm{OH})_{2} \mathrm{TBC}(\mathrm{OiPr})_{4}\right\}$ dihydroxy phosphorus $(\mathrm{V})$ tetrabenzotriazacorroles (the structures are shown in Figure 9) measured by femtosecond (50 fs) degenerate four-wave mixing technique (Huang et al., 2008).

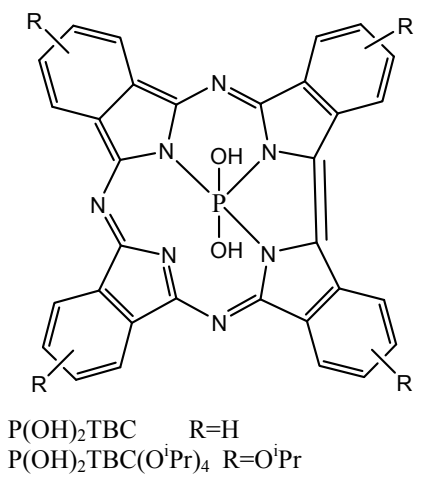

(a)

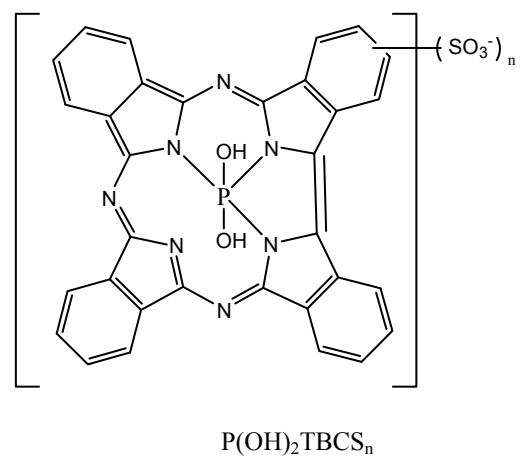

(b)

Fig. 9. Structural formulae of (a) $\mathrm{P}(\mathrm{OH})_{2} \mathrm{TBC}$ and $\mathrm{P}(\mathrm{OH})_{2} \mathrm{TBC}(\mathrm{OiPr})_{4} ;(\mathbf{b}) \mathrm{P}(\mathrm{OH})_{2} \mathrm{TBCSn}$.

The evaluated values of $\chi(3)$ and $\gamma$ for these phthalocyanine analogues are summarized in Table 3 together with the visible absorption maxima $\left(\lambda_{\max }\right)$ and nonlinear refractive index in DMF solutions. The $\gamma$ of samples show high values as large as $10^{-31} \mathrm{esu}$, and the highest value of $2.26 \times 10^{-31}$ esu for the $\mathrm{P}(\mathrm{OH})_{2} \mathrm{TBC}(\mathrm{O} \mathrm{Pr})_{4}$ was observed, which is almost two times larger than that of phthalocyanine analogue $\mathrm{P}(\mathrm{OH})^{2} \mathrm{TBC}$. This is probably due to the isopropoxyl group is attached to the benzene rings of the phthalocyanine analogue $\mathrm{P}(\mathrm{OH})_{2} \mathrm{TBC}(\mathrm{OiPr})_{4}$, and isopropoxyl group is a electron-donating group which leads to large polarization of molecules. Considering molecular structure of invested phthalocyanine analogues, two hydroxyl moieties complexed with central phosphorus of molecule in axial direction, and form a three-dimensional configuration, which can enhance third-order optical nonlinearity of molecule. Moreover, their response times are also obtained and no more than $50 \mathrm{fs}$, which are commonly accepted to the contribution from the transient motion of the conjugate electron distribution.

\begin{tabular}{ccccc}
\hline Sample & $\lambda_{\max }(\mathrm{nm})$ & $\chi^{(3)}\left(\times 10^{-14} \mathrm{esu}\right)$ & $\begin{array}{c}\gamma \\
\left(\times 10^{-14} \mathrm{esu}\right)\end{array}$ & $n_{2}\left(\times 10^{-14} \mathrm{esu}\right)$ \\
\hline $\mathrm{P}(\mathrm{OH})_{2} \mathrm{TBC}$ & 655 & 4.40 & 1.21 & 8.15 \\
$\mathrm{P}(\mathrm{OH})_{2} \mathrm{TBCSn}$ & 657 & 4.27 & 1.11 & 7.98 \\
$\mathrm{P}(\mathrm{OH})^{2} \mathrm{TBC}(\mathrm{OiPr})_{4}$ & 677 & 4.91 & 2.26 & 9.01 \\
\hline
\end{tabular}

Table 3. The evaluated values of $\chi^{(3)}, \gamma$, and $n_{2}$ for the dihydroxy phosphorus (V) tetrabenzotriazacorroles together with the visible absorption maxima $\left(\lambda_{\max }\right)$ in DMF solution. 


\subsubsection{Rare earth polymeric phthalocyanines}

In 2008, we (Zhao et al., 2008) have synthesized three novel tri-dimensional phthalocyanine polymers with lanthanum ( $\mathrm{LaPPc}$ ), gadolinium (GdPPc) and ytterbium (YbPPc) as centric atoms from a tetranuclear phthalonitrile (the structures and synthesis route of rare earth polymeric phthalocyanines are shown in Scheme 3). And third-order optical nonlinearities of these compounds in DMF solution were measured by a picosecond Z-sacn technique at $532 \mathrm{~nm}$.

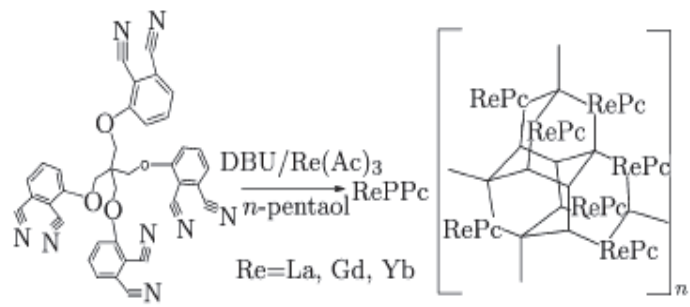

Scheme 3. Structures and synthesis route of rare earth polymeric phthalocyanines

Based on the Z-scan measurements, it is found that these phthalocyanine polymers show large third-order nonlinear susceptibilities which are of the order of $10^{-12}$ esu. Both the nonlinear absorption $\beta$ and nonlinear refraction $n_{2}$ decreases with the order of LaPPc $>$ GdPPc $>$ YbPPc. For all the compounds, the values of $\operatorname{Re} \chi^{(3)}$ are one order of magnitude larger than those of $\operatorname{Im} \chi^{(3)}$, which determine the magnitude of third-order nonlinear susceptibilities $\chi^{(3)}$. This indicated that the nonlinear refraction is predominant mechanism for the nonlinear optical response of three phthalocyanine polymers.

Furthermore, Researchers (Manas et al., 1997) have described the effect of intermacrocycle interactions on the second hyperpolarizabilities of phthalocyanine dimer and trimer. It was considered that stack form of phthalocyanine can induce molecular electronic interaction between neighbouring phthalocyanine rings and induce charge transfer between them. As shown in Figure 10, the cage structure of phthalocyanine polymer presents more possibilities of electronic interaction and distortion of electron cloud in three dimensions at intermolecular scale. This would elevate the spatial polarizability of molecular and induces large nonlinear coefficient. However, the trimer or binuclear phthalocyanine could only offer this intermolecular possibility from two dimensions.

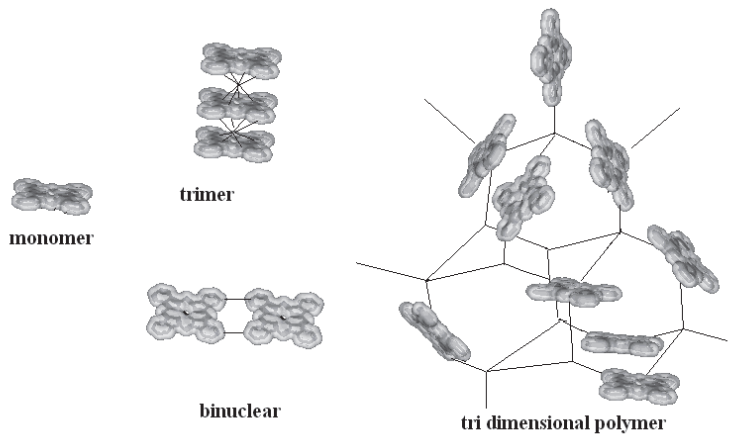

Fig. 10. Calculated molecular electronic distribution of binuclear, trimer and polymer of phthalocyanine. 


\section{Conclusions}

Major advances have been made in the design and synthesis of the novel phthalocyanines and related compounds for third-order optical nonlinearity. They provide useful examples to illustrate the new features of the NLO response of the phthalocyanines and related compounds. This review summarizes our results recently obtained on the correlation between molecular structure and NLO response and offers some strategies for rendering new systems with improved NLO properties.

Owing to the extended $\pi$ system, it is well-known that these Pc-related compounds exhibit a high aggregation tendency, and the aggregates usually display outstanding nonlinear optical properties. Moreover, by introducing peripheral substituents or by adding axial substituents, one may obtain three-dimensional (3D) structures with pyramidal shape and examine the effect of a third-dimension on the NLO response. Some of the four isoindole units can be formally replaced by other heterocyclic moieties, giving rise to different phthalocyanine analogues. All these chemical variations can alter the electronic structure of the macrocyclic core, and therefore, they allow the fine-tuning of the nonlinear response.

In conclusion, one could say that structural variations explored in phthalocyanines, such as metal insertion, introduction of functional groups into the periphery of the macrocycle, extension of conjugation, and variation of the main structure of the macrocycle, allow the tuning of the nonlinear responses. However, a detailed understanding of the factors affecting the nonlinear response is still necessary, and further work should be devoted to this objective.

\section{Acknowledgments}

We appreciate the help of Dr. Li Deng and Professor Zhenrong Sun, Key Laboratory of Optical and Magnetic Resonance Spectroscopy, East China Normal University, in carrying out the DFWM experiment. This work was supported by National Natural Science General Foundation of China (Grants 20773077, 20572059 and 20502013) and National Key Fundamental Research Program (2007CB808000).

\section{References}

Perry, J.W.; Mansour, K.; Mander, K.J.; Perry, K.J.; Alvarez, D. \& Choong, I. (1994). Enhanced Reverse Saturable Absorption and Optical Limiting in Heavy-AtomSubstituted Phthalocyanines, Optical Letters, Vol.19,PP.625-627.

Perry, J.W.; Mansour, K.; Lee, I.-Y.S. \& Wu, X.-L. (1996). Organic Optical Limiter with a Strong Nonlinear Absorptive Response, Science, Vol.273,PP.1533-1536.

Shirk, J.S.; Pong, R.F.S.; Flom, S.R.; Heckmann, H. \& Hanack, M. (2000). Effect of Axial Substitution on the Optical Limiting Properties of Indium Phthalocyanines, Journal of Physical Chemistry A, Vol.104,PP.1438-1449.

Zyss, J. (Ed.). (1994). Molecular Nonlinear Optics: Materials, phisics and Devices, Academic Press, New York.

Ho, Z.Z.; Ju, C.Y. \& Hetheringto, W.M. (1987). Third Harmonic Generation in Phthalocyanines, Journal of Applied physics, Vol.62, PP.716-718. 
Shirk, J.S.; Lindle, F.J.; Bartoli, C.A.; Hoffman, C.A.; Kafafi, Z.H. \& Snow, A.W. (1989). OffResonant Third-Order Optical Nonlinearities of Metal-Substituted Phthalocyanines, Applied Phisics Letters, Vol.55, PP.1287-1288.

Kambara, H.; Muruno, T.; Yamashita, A.; Matsumoto, S.; Hayashi, T.; Konami, H. \& Tanaka, N. (1996). Third-Order Nonlinear Optical Properties of Phthalocyanine and Fullerene, Journal of Applied Physics, Vol.80, PP.3674-3682.

Ma, g.; He, J.; Kang, C. \& Tang, S. (2003). Excited State Dynamics Studies of Iron(III) Phthalocyanine Using Femtosecond Pump-Probe Techniques, Chemical Physics Letters, Vol.370, PP.293-299.

Zhou, J.; Mi, J.; Zhu, R.; Li, B. \& Qian, S. (2004). Ultrafast Excitation Relaxation in Titanylphthalocyanine Thin Film, Optical Materials, Vol.27, PP.377-382.

He, C.; Wu, Y.; Shi, G.; Duan, W.; Song, W. \& Song, Y. (2007). Large Third-Order Optical Nonlinearites of Ultrathin Films Containing Octacarboxylic Copper Phthalocyanine, Organic Electronics, Vol.8, PP.198-205.

Hermann, J.P.; Ricard, D. \& Ducuing, J. (1973). Optical Nonlinearities in Conjugated Systems: $\beta$-Carotene, Applied Physics Letters, Vol.23, PP.178-180.

Linder, R.C.; Steel, D.G. \& Dunning, G.J. (1982). Phase Conjugation by Resonantly Enhanced Degenerate Four-Wave Mixing, Optical Engineering, Vol.21, PP.190-198.

Sheik-Bahae, M.; Said, A.A. \& van Stryland, E.W. (1989). High-Sensitivity, Single-Beam $\mathrm{n}_{2}$ Measurements, Optical Letters, Vol.14, PP.955-957.

Sheik-Bahae, M.; Said, A.A.; Wei, T.H.; Hagan, D.J. \& van Stryland, E.W. (1990). Sensitive Measurement of Optical Nonlinearities Using a Single Beam, IEEE Journal of Quantum Electronics, Vol.26, PP.760-769.

Torre, G.; Vazquez, P.; Agullo-Lopez, F. \& Torres, T. (2004). Role of Structure Facror in the Nonlinear Optical Properties of Phthalocyanines and Related Compounds, Chemical Reviews, Vol.104, PP.3723-3750.

Nalwa, H.S.; Kakuta, A. \& Mukoh, A. (1993). Third-Order Optical Nonlinearities of Tetrakisn-Pentoxy Carbonyl Metallo-Naphthalocyanines, Chemical Physics Letters, Vol.203, PP.109-113.

Nalwa, H.S.; Hanack, M.; Pawlowski, G. \& Klaus, E.M. (1999). Third-Order Nonlinear Optical Properties of Porphyrazine, Phthalocyanine and Naphthalocyanine Germanium Derivatives: Demonstrating the Effect of $\pi$-Conjugation Length on Third-Order Optical Nonlinearity of Two-Dimensional Molecules, Chemical Physics, Vol.245, PP.17-26.

He, C.; Chen, Y.; Nie, Y. \& Wang, D. (2007). Third Order Optical Nonlinearities of Eight- $\beta$ Octyloxy-Phthalocyanines, Optics Communications, Vol.271, PP.253-256.

Li, Z.; Chen, Z.; Xu, S.; Niu, L.; Zhang Z.; Zhang, F. \& Kasatni, K. (2007). Off-Rsonant ThirdOrder Optical Nonlinearities of Novel Diarylethene-Phthalocyanine Dyads, Chemical Physics Letters, Vol.447, PP.110-114.

Chen, Z.; Zhong, C.; Zhang, Z.; Li, Z.; Niu, L.; Bin, Y. \& Zhang, F. (2008). Photoresponsive JAggregation Behavior of a Novel Azobenzene-Phthalocyanine Dyad and Its ThirdOrder Optical Nonlinearity, Journal of Physical Chemistry B, Vol.112, PP.7387-7394.

Huang, X.; Zhao, F.; Li, Z.; Tang, Y.; Zhang, F. \& Tung, C.-H. (2007). Self-Assembled Nanowire Networks of Aryloxy Zinc Phthalocyanines Based on Zn-O Coordination, Langmuir, Vol.23, PP.5167-5172. 
Huang, X.; Zhao, F.; Li, Z.; Huang, L.; Tang, Y.; Zhang, F. \& Tung, C.-H. (2007). A Novel Self-Aggregates of Phthalocyanine Based on Zn-O Coordination, Chemistry Letters, Vol.36, PP.108-109.

Chen, Z.; Dong, S.; Zhong, C.; Zhang, Z.; Niu, L.; Li, Z. \& Zhang, F. (2009). Photoswitching of Third-Order Optical Properties of Azobenzene-Containing Phthalocyanines Based on Reversible Host-Guest Interactions, Journal of Photochemistry and Photobiology A: Chemistry, Vol.206, PP.213-219.

Breslow, R. \& Dong, S. (1998). Biomimetic Reactions Catalyzed by Cyclodextrins and Their Derivatives, Chemical Review, Vol.98, PP.1997-2012.

Dugave, C. \& Demange, L. (2003). Cis-Trans Isomerization of Organic Molecules and Biomolecules: Implications and Applications, Chemical Review, Vol.103, PP.24752532.

González-Cabello, A.; Claessens C, G.; Martin-Fuch, G.; Ledoux-Rack, I.; Vázquez, P.; Zyss, J.; Agulló-López, F. \& Torres, T. (2003). Phthalocyanine-Ferrocene Dyads and Triads for Nonlinear Optics, Synthsis Metal, Vol. 137, PP.1487-1488.

Bin, Y.; Xu, S.; Li, Z.; Huang, L.; Zhang, Z. \& Zhang, F. (2008). Large Third-Order Optical Nonlinearity of a Novel Copper Phthalocyanine Ferrocene, Chinese Physics Letters, Vol.25, PP.3257-3259.

Liu, J.; Zhang, F. ; Zhao, Y ; Tang, Y ; Song, X. \& Yao, G. (1995). Complexation of Phosphorus (III) with a Novel Tetrapyrrolic Phthalocyanine-Like Macrocyclic Compound, Journal of Photochemistry and Photobiology A: Chemistry, Vol.91, PP.99-104.

Chen, Z.; Zhou, X.; Li, Z.; Niu, L.; Yi, J. \& Zhang, F. (2011). The Third-Order Optical nonlinearities of Thiophene-Bearing Phthalocyanines Studied by Z-Scan Technique, Journal of Photochemistry and Photobiology A: Chemistry, Vol.211, PP.64-68.

Huang, L. Li, Z.; Zhang, F.; Tung, C. \& Kasatani, K. (2008). Off-Resonant Optical Nonlinearities of Phthalocyanine Analogues: Dihydroxy Phosphorus (V) Tetrabenzotriazacorroles, Optics Communication, Vol.281, PP.1275-1279.

Zhao, P.; Xu, S.; Li, Z. \& Zhang, F. (2008). Nonlinear Optical Properties of Novel Polymeric Rare Earth Phthalocyanine Studied Using Picosecond Z-scan Technique, Chinese Physics Letters, Vol.6, PP.2058-2061.

Manas, E.S; Spano, F.C \& Chen, L.X. (1997). Nonlinear Optical Response of Cofacial Phthalocyanine Dimers and Trimers, Journal of Chemical Physics, Vol.107, PP.707719. 


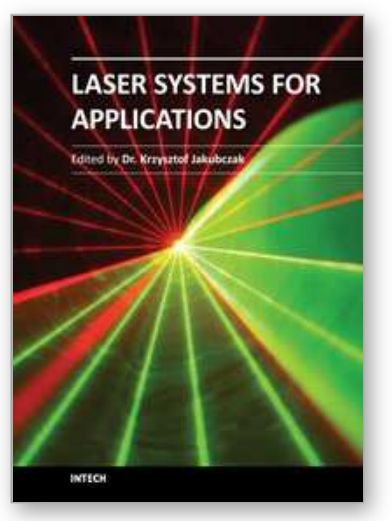

\author{
Laser Systems for Applications \\ Edited by Dr Krzysztof Jakubczak
}

ISBN 978-953-307-429-0

Hard cover, 308 pages

Publisher InTech

Published online 14, December, 2011

Published in print edition December, 2011

This book addresses topics related to various laser systems intended for the applications in science and various industries. Some of them are very recent achievements in laser physics (e.g. laser pulse cleaning), while others face their renaissance in industrial applications (e.g. $\mathrm{CO} 2$ lasers). This book has been divided into four different sections: (1) Laser and terahertz sources, (2) Laser beam manipulation, (3) Intense pulse propagation phenomena, and (4) Metrology. The book addresses such topics like: Q-switching, mode-locking, various laser systems, terahertz source driven by lasers, micro-lasers, fiber lasers, pulse and beam shaping techniques, pulse contrast metrology, and improvement techniques. This book is a great starting point for newcomers to laser physics.

\title{
How to reference
}

In order to correctly reference this scholarly work, feel free to copy and paste the following:

Zhongyu Li, Zihui Chen, Song Xu, Xinyu Zhou and Fushi Zhang (2011). Third-Order Optical Nonlinearities of Novel Phthalocyanines and Related Compounds, Laser Systems for Applications, Dr Krzysztof Jakubczak (Ed.), ISBN: 978-953-307-429-0, InTech, Available from: http://www.intechopen.com/books/laser-systems-forapplications/third-order-optical-nonlinearities-of-novel-phthalocyanines-and-related-compounds

\section{INTECH}

open science | open minds

\section{InTech Europe}

University Campus STeP Ri

Slavka Krautzeka 83/A

51000 Rijeka, Croatia

Phone: +385 (51) 770447

Fax: +385 (51) 686166

www.intechopen.com

\section{InTech China}

Unit 405, Office Block, Hotel Equatorial Shanghai

No.65, Yan An Road (West), Shanghai, 200040, China

中国上海市延安西路65号上海国际贵都大饭店办公楼 405 单元

Phone: +86-21-62489820

Fax: +86-21-62489821 
(C) 2011 The Author(s). Licensee IntechOpen. This is an open access article distributed under the terms of the Creative Commons Attribution 3.0 License, which permits unrestricted use, distribution, and reproduction in any medium, provided the original work is properly cited. 